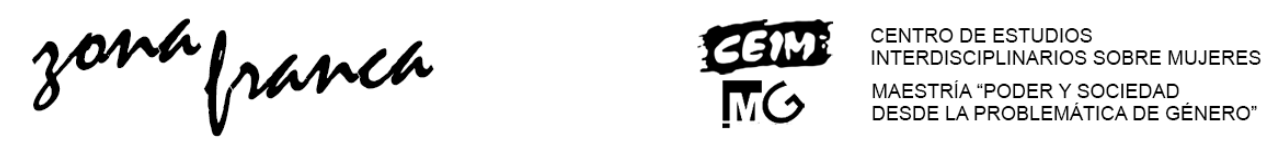

\title{
El género de la gestión pública federal brasileña': Mujeres en las carreras de la gestión gubernamental (1995-2017)
}

\author{
Elaine de Melo Xavier ${ }^{*}$ Silvia Cristina Yannoulas **
}

\section{Resumen}

Los procesos de producción y reproducción de las desigualdades sociales que estructuran la sociedad brasileña continúan restringiendo las posibilidades de competir por un empleo público a los grupos que no reúnen condiciones para aprobación de concurso público (establecido en la Constitución Federal de 1988 para ocupación de cargos en la administración pública). También determinan los nichos que están abiertos para quien logra ultrapasar las barreras iniciales. En el

'En este artículo presentamos resultados de investigación de maestría realizada en el contexto del Programa de Post-grado en Política Social de la UnB, elaborada por Elaine de Melo Xavier bajo la dirección de Silvia Cristina Yannoulas. Corresponden al capítulo 4 del trabajo final defendido en diciembre de 2018. El proyecto de investigación fue previamente aprobado por comité de ética en investigación, vía Plataforma Brasil (http://plataformabrasil.saude.gov.br), en 06/03/2018 (proceso 83321417.1.0000.5540).

Ministerio de Planificación, Desarrollo y Gestión, Brazil. Formada en Relaciones Internacionales por el Centro Universitario de Brasília. Especialista en Relaciones Internacionales por la Universidad de Brasília y Master en Política Social por la Universidad de Brasilia. Analista de Planeamiento y Presupuesto. Miembro del Grupo de Investigación Trabajo, Educación y Discriminación. Contacto: elaine.xavier@ibest.com.br

** Universidad de Brasília, Brazil. Licenciada en Ciencias de la Educación por la Universidad de Buenos Aires, Master en Ciencias Sociales por la Facultad Latinoamericana de Ciencias Sociales Sede Académica Argentina, Doctora en Sociología por la Universidad de Brasília, Post-Doctora en Educación por la Universidad Federal de Minas Gerais. Profesora del Departamento de Trabajo Social y del Programa de Post-grado en Política Social de la Universidad de Brasília. Líder del Grupo de Investigación Trabajo, Educación y Discriminación. Contacto: silviayannoulas@unb.br

de Melo Xavier, Elaine; Yannoulas, Silvia Cristina. "El género de la gestión pública federal brasileña: Mujeres en las carreras de la gestión gubernamental (1995-2017)" en Zona Franca. Revista del Centro de estudios Interdisciplinario sobre las Mujeres, y de la Maestría poder y sociedad desde la problemática de Género, №27, 2019 pp.218-239. ISSN, 2545-6504 Recibido: 29 de enero 2019; Aceptado: 22 de noviembre 2019

Revista Zona Franca- Centro de estudios interdisciplinario sobre las mujeres (CEIM)- Maestría poder y sociedad desde la problemática de género (MG), Rosario, Argentina. ISSN, 2545-6504 http://zonafranca.unr.edu.ar/index.php/ZonaFrancal Numero 27 (2019). 
caso de las mujeres, el empleo público históricamente tuvo relevancia porque otorga garantías para el ejercicio del maternaje, pero las restringió a espacios específicos en las áreas sociales, y aun así sin ocupar cargos relevantes. La investigación que realizamos mostró que ellas están en áreas consideradas no estratégicas, más expuestas al proceso de precarización, y que enfrentan dificultades específicas para la promoción dentro de la carrera administrativa. Las 14 personas entrevistadas nos ayudaron a comprender los mecanismos de discriminación encubiertos.

Palabras claves: División sexual del trabajo - Sector Público Carreras - Políticas Públicas - Brasil.

\section{The gender in the Brazilian federal public management: Women in the} careers of government management (1995-2017)

\section{Abstract}

The processes of production and reproduction of social inequalities that structure Brazilian society continue to restrict the possibilities to compete for public employment to groups that do not meet the conditions for approval of public contest (established in the 1988 Federal Constitution for occupation of positions in public administration). They also determine the niches that are open for those who manage to surpass the initial barriers. In the case of women, public employment historically had relevance because it provides guarantees for the exercise of mothering, but restricted them to specific spaces in social areas, and even then without occupying relevant positions. The research we conducted showed that they are in non-strategic considered areas, more exposed to the precarization process, and that they also face specific difficulties for promotion within the administrative career. The 14 people interviewed helped us understand the covert mechanisms of discrimination.

Key words: Sexual division of labor - Public sector - Careers Public policy - Brazil.

\section{Introducción}

En un mercado de trabajo formal en proceso de reducción y precarización, la administración pública federal representa una de las mejores alternativas de empleo para las y los trabajadores brasileños. Hasta la década de 1970-1980, según Barroso (2015), el empleo público era considerado una alternativa poco deseable para aquellos trabajadores con diploma de educación superior, y atraía

Revista Zona Franca- Centro de estudios interdisciplinario sobre las mujeres (CEIM)- Maestría poder y sociedad desde la problemática de género (MG), Rosario, Argentina. ISSN, 2545-6504 http://zonafranca.unr.edu.ar/index.php/ZonaFranca| Numero 27 (2019). 
apenas a aquellos y aquellas que tenían dificultades para participar del sector privado del mercado de trabajo. Sin embargo, a partir de la década de 1980-1990 y con el proceso de reestructuración productiva en curso, el empleo público federal se transformó en el "sueño dorado" de los trabajadores con mayor nivel de escolaridad.

Reconocer la mejor calidad del empleo público frente al privado en la actualidad no significa afirmar que el proceso de precarización no alcanzó a las condiciones de trabajo en el Estado, o que todos los empleados públicos tengan condiciones equivalentes de ingreso y promoción interna. Entre 1995 y 2017, los procesos sociales de producción y reproducción de las desigualdades de clase, sexo/género y raza/etnia que estructuran la sociedad brasileña continuaron a restringir las posibilidades de competición por un empleo público a ciertos grupos sociales (no propietarios, mujeres, negros), que no reúnen las condiciones objetivas para aprobación en concurso público (establecido en el Brasil en la Constitución de 1988 para ocupación de cargos administrativos), y también siguen determinando los espacios dentro de la administración pública abiertos para aquellos que consiguen ultrapasar las barreras iniciales.

Para las mujeres, el empleo público siempre tuvo mucha relevancia, porque permite garantías específicas (licencias, horarios especiales, etc.) para el ejercicio de maternaje. La investigación que realizamos confirmó que ellas se incorporan preferencialmente en áreas consideradas no estratégicas por el Plan Director de la Reforma de Estado - PDRAE (BRASIL 1995), y por lo tanto, áreas más expuestas al proceso de precarización.

Aún aquellas empleadas públicas incluidas en el denominado núcleo estratégico, como las integrantes de las carreras de la gestión gubernamental federal - enfrentan dificultades para estar en pie de igualdad frente a sus pares 
hombres, porque no logran ocupar cargos de alto nivel decisorio". Se registra poca participación de las mujeres en los cargos de nivel superior de las carreras de la gestión gubernamental, y también su menor participación en cargos comisionados del mayor nivel de decisión en la administración pública federal brasileña (BRASIL 2017; BRASIL 2016a).

Es decir, la segmentación horizontal (áreas sociales próximas a la reproducción social) y vertical (no ocupación de altos cargos, inclusive en las áreas sociales donde las mujeres predominan), relatada por Yannoulas (2003) para el mercado de trabajo, se manifiesta hasta los días de hoy al interior de la administración pública federal brasileña. Podemos afirmar que hubo un proceso de feminilización de la administración pública, con un ingreso paulatinamente significativo de mujeres que acompañó la implementación de los concursos públicos para la ocupación de los cargos desde 1988, pero todavía no hay equiparación entre hombres y mujeres cuanto a su distribución en las áreas o políticas sectoriales, y tampoco hubo un proceso de feminización de la gestión pública que les permita acceder a los más altos cargos de la carrera"'.

Para comprender de qué manera el sexo/género interfiere en la trayectoria profesional de hombres y mujeres de las carreras de la gestión gubernamental

"En el PDRAE, el "núcleo estratégico" es el responsable por la definición de las leyes y políticas públicas (BRASIL 1995). Además del núcleo estratégico, el aparato de Estado seria constituido por otros 3 segmentos: (1) el sector de "actividades exclusivas del Estado", con servicios exclusivamente a cargo del Estado; (2) el sector de "servicios no-exclusivos", en los cuales el Estado actúa simultáneamente con organizaciones públicas no estatales, y referidos a derechos fundamentales; y (3) el sector de "producción de bienes y servicios para el mercado", caracterizado por las actividades económicas dirigidas al lucro dentro del aparato de Estado (BRASIL 1995).

III Segundo Yannoulas (2013), en la literatura especializada sobre género y trabajo son utilizados alternativamente dos significados distintos para la categoría feminização de las profesiones y ocupaciones, que se corresponden con metodologías y técnicas diferentes para colectar y analizar la información pertinente. Un significado cuantitativo, feminilização, referido al aumento del peso relativo del sexo femenino en la composición de una profesión o ocupación; su medición y análisis son realizadas a través de datos estadísticos; y un significado cualitativo, feminização, alude a las transformaciones de significado y valor social de una profesión o ocupación, originadas a partir de la feminilização y relacionadas a los conceptos de género predominantes en un determinado período histórico; su impacto puede observarse a través del análisis de discurso. Hay una intensa relación entre ambos conceptos.

Revista Zona Franca- Centro de estudios interdisciplinario sobre las mujeres (CEIM)- Maestría poder y sociedad desde la problemática de género (MG), Rosario, Argentina. ISSN, 2545-6504 http://zonafranca.unr.edu.ar/index.php/ZonaFranca| Numero 27 (2019). 
federal brasileña, fueron realizadas en 2018 entrevistas semi-estructuradas con 14 empleados públicos federales de dos cargos de nivel superior: los cargos efectivos de Analista de Planeamiento y Presupuesto - APO ${ }^{\mathrm{IV}}$, y de Especialista en Políticas y Gestión Gubernamental - EPPGG.

La participación de los entrevistados fue voluntaria y la selección fue realizada por medio de la técnica "bola de nieve", con referencias realizadas por cada entrevistado sobre otros potenciales entrevistados, para construir así una muestra no-probabilística de hombres y mujeres de la administración pública federal directa, APO y EPPGG que ocupan u ocuparon recientemente una Función Comisionada en el Poder Ejecutivo - FCPE, de nivel 4, y/o cargo en comisión de

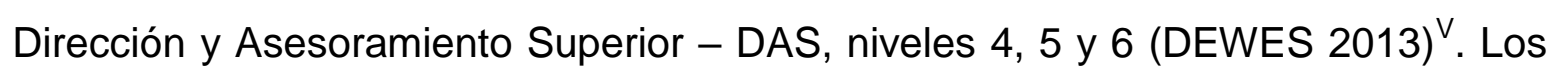
ocupantes de tales cargos comisionados representan, juntamente con los ocupantes de cargos de Naturaleza Especial - NES, como Presidente da República, Ministros de Estado o Secretarios Ejecutivos, la elite dirigente del País.

Una vez realizadas, las entrevistas fueron desgravadas y organizadas en dos grandes grupos: concedidas por hombres y por mujeres. Cada uno de los bloques fue analizado a partir de una lectura horizontal seguida de otra transversal, conforme sugerido por Minayo (2008).

El artículo está organizado en tres partes: la primera presenta el perfil de los APO y EPPGG entrevistados; la segunda muestra la interferencia del sexo/género en la trayectoria profesional de los y las entrevistadas; y la tercera parte concluye sobre la inserción de las mujeres en el aparato estatal brasileño.

\section{EI Perfil de APO y EPPGG Entrevistados/as}

\footnotetext{
IV Presupuesto en portugués es orçamento, por eso la sigla es APO.

$\checkmark$ De acuerdo con la Ley $n^{\circ}$ 13.346, de 10 de octubre de 2016, la diferencia entre los cargos DAS y las FCPE está en quién está apto para su ocupación. La FCPE solamente puede ser ocupada por servidores públicos efectivos (concursados).
}

Revista Zona Franca- Centro de estudios interdisciplinario sobre las mujeres (CEIM)- Maestría poder y sociedad desde la problemática de género (MG), Rosario, Argentina. ISSN, 2545-6504 http://zonafranca.unr.edu.ar/index.php/ZonaFranca| Numero 27 (2019). 
Los 14 APO y EPPGG entrevistados/as en 2018 eran en su gran mayoría blancos, graduados o post-graduados en Economía, entre los 41 y los 50 años, casados y con 2 hijos. Existían 3 diferencias importantes entre hombres y mujeres, que merecen ser comentadas aquí.

En primer lugar, apenas los hombres ocupan cargos más elevados (DAS 6), siendo el cargo más alto ocupado por mujeres DAS 5.

En segundo lugar, el grupo de las mujeres APO y EPPGG registró mayor diversidad étnico/racial que el grupo de los hombres, con mayor participación de mujeres entrevistadas auto-declaradas negras y pardas.

Tercero, a pesar de la graduación en Ciencias Económicas ser la más frecuente tanto entre ellas como entre ellos, se registró mayor diversidad de áreas de formación entre ellas. Así, mientras los graduados en Economía representan $71,4 \%$ de los entrevistados; las graduadas en Economía constituían apenas 42,9\% de las entrevistadas.

Los datos relativos al post-grado también presentan diferencias por sexo/género. 100\% de los hombres APO e EPPGG entrevistados realizó algún post-grado (lato sensu e/o stricto sensu), e apenas $71,4 \%$ de las entrevistadas. Entre ellos, dos áreas del conocimiento concentran gran parte de los post-grados realizados: Economía (42,8\%) y Administración Pública (también 42,8\%). Entre las mujeres APO y EPPGG entrevistadas, las áreas de conocimiento de los postgrados realizados eran variadas, desde Energía hasta Desarrollo Internacional, siendo solamente 14,3\% en Economía y ninguna en Administración Pública. Además, los post-grados lato sensu (de corta duración y más especializados) eran más comunes entre ellas.

Otra diferencia significativa entre entrevistados y entrevistadas se relaciona con el lugar de inserción: mientras que los entrevistados se concentraron en el Ministerio de Planeamiento, Presupuesto y Gestión, las mujeres estaban dispersas

Revista Zona Franca- Centro de estudios interdisciplinario sobre las mujeres (CEIM)- Maestría poder y sociedad desde la problemática de género (MG), Rosario, Argentina. ISSN, 2545-6504 http://zonafranca.unr.edu.ar/index.php/ZonaFrancal Numero 27 (2019). 
en diferentes órganos de la administración pública federal directa. Además, la edad media de los hijos de los entrevistados era de 9 años y de 13 años en el caso de las entrevistadas, lo que indicaría que ellas tuvieron que esperar más tiempo para ocupar cargos altos después del nacimiento de los hijos.

Los dos grupos presentan características semejantes cuanto a: (1) salario básico bruto ${ }^{\mathrm{VI}}$ medio de ellos $\mathrm{R} \$ 31.404,68$ y de ellas $\mathrm{R} \$ 31.846,55$; (2) tiempo medio de carrera de ellos 13 años y de ellas 15 años.

\section{Sexo/género en la Trayectoria Profesional de los y las Entrevistadas}

La división sexual del trabajo, conforme destacado por Yannoulas (2003), posee bases materiales y bases simbólicas, que se reproducen y fortalecen mutuamente. De esta manera, no es por acaso que el grupo de los entrevistados percibía hombres y mujeres como seres ontológicamente diferentes, y que por lo tanto sus inserciones diferenciadas en la administración pública no fuesen, en general, problematizadas.

Entre los entrevistados, las características atribuidas a los géneros se relacionaron con los espacios prioritarios de actuación de cada grupo social. Las mujeres, para 4 de los entrevistados, podían caracterizarse como "comunicativas" y "conciliadoras", mientras que los hombres serían "objetivos", "competitivos" y "dispuestos a la tarea". Frente a esta percepción, que afirma una diferencia esencial entre hombres y mujeres, parece natural que las mujeres se interesen por determinadas áreas del conocimiento y posteriormente de inserción laboral.

Entre las entrevistadas, las mujeres también fueron definidas como "poco objetivas", más "honestas", "organizadas", “dóciles", "sensibles", "informales",

\footnotetext{
${ }^{V I}$ La información sobre los salarios tomó como referencia el mes de febrero de 2018, primer mes disponible para consulta en el Portal de la Transparencia al momento de nuestra investigación (Disponible en: www.portaldatransparencia.gov.br. Acceso en 21 septiembre de 2018). La remuneración básica bruta es compuesta por la suma de los pagos por cargo efectivo, función o cargo comisionado.
}

Revista Zona Franca- Centro de estudios interdisciplinario sobre las mujeres (CEIM)- Maestría poder y sociedad desde la problemática de género (MG), Rosario, Argentina. ISSN, 2545-6504 http://zonafranca.unr.edu.ar/index.php/ZonaFranca| Numero 27 (2019). 
"colaborativas", "responsables", "altruistas", "comunicativas", "con mayor capacidad de adaptación" y "más ligadas a la familia". Ya los hombres fueron descritos como "objetivos", "ambiciosos", "vanidosos", "formales", "duros", "ríspidos", "más sueltos" y "más focalizados en la tarea". Lo interesante en el discurso de ellas fue percibir una especie de "espejo" en las características atribuidas a mujeres y hombres (objetivo $X$ poco objetiva, formal $X$ informal, ríspido $X$ dócil, entre otros). Además, se establece una relación de complementariedad, en el sentido de que las características atribuidas a cada grupo fueron descritas como necesarias para que puedan actuar en los espacios designados. De esta manera, el discurso refuerza que las características de las mujeres las hacen más aptas para las actividades reproductivas en espacios privados, en los cuales las relaciones son construidas por afecto, cuidado y apoyo mutuo. Los hombres serían más apropiados para el espacio público, en el cual las relaciones son estructuradas alrededor de la competición.

En 3 de las mujeres entrevistadas la colonización mental producto de la ideología patriarcal encontraba resistencia (LÉON 2002). Para ellas, las diferencias apuntadas no solamente no eran reales, sino que eran señaladas como resultado de la vigencia de un padrón de opresión sexista.

Mostramos a las entrevistadas los datos sobre la participación de las mujeres en las carreras de gestión gubernamental divulgados por el Boletín Estadístico de Personal e Informaciones Organizacionales - BEP no 249 (BRASIL 2017) ${ }^{\mathrm{VII}}$, y preguntamos sobre los factores que podrían explicar la desigualdad entre hombres y mujeres. Las entrevistadas se dividieron en dos grupos: las que piensan que la prueba de selección para ocupar cargos no favorece el ingreso de mujeres, por su impronta economicista, y aquellas que no identifican motivos especiales para

VII El BEP no 249 (BRASIL 2017) mostró una participación de las mujeres de solo el $31.4 \%$ en las carreras de la gestión gubernamental. Entre los APO, ellas representaban el $26,7 \%$ de los servidores y entre los EPPGG, el 35,0\%. En los cargos y funciones comisionados, las mujeres representaban solo el 34,3\%, 22,5\% y 18,6\% de los ocupantes de FCPE / DAS 4, DAS 5 y DAS 6 , respectivamente, mientras que su participación en el servicio público federal en el mismo año alcanzó $46,4 \%$.

Revista Zona Franca- Centro de estudios interdisciplinario sobre las mujeres (CEIM)- Maestría poder y sociedad desde la problemática de género (MG), Rosario, Argentina. ISSN, 2545-6504 http://zonafranca.unr.edu.ar/index.php/ZonaFrancal Numero 27 (2019). 
explicar la situación desigual, siendo este último grupo constituido apenas por entrevistadas EPPGG. Una de las entrevistadas que destacó la impronta de la prueba como causa de la desigualdad ilustró bastante bien cómo el "imaginario de género" colabora para la manutención de la segmentación horizontal de la administración pública federal. En su discurso se establecía una intrínseca relación entre "lo femenino", las áreas de formación educacional con mayor adherencia, y las salidas profesionales a partir de las elecciones realizadas por las mujeres al optar por ciertos carreras ${ }^{\mathrm{VIII}}$.

Los entrevistados, cuando confrontados con los datos oficiales registrados por el mencionado boletín (BRASIL 2017), ofrecieron un abanico más amplio de respuestas explicativas de la diferencia entre hombres y mujeres en las carreras de la gestión gubernamental: (1) el "tipo de asunto" tratado por esas carreras, que no sería atractivo para las mujeres; (2) las desigualdades de sexo/género heredadas del pasado; (3) el perfil de la prueba de selección, que no privilegia las áreas de conocimiento preferidas por las mujeres; y (4) la menor disponibilidad de tiempo de las mujeres para estudiar para concursos públicos difíciles y concurridos por las actividades de maternaje. También entre los entrevistados fue notable la relación establecida entre "lo femenino", la formación educacional y las salidas laborales según la elección anterior.

Sin embargo, la relación establecida por diversos entrevistados (hombres y mujeres) entre la menor presencia de las mujeres en los cursos de Economía y la pequeña participación de ellas en cargos de APO y EPPGG debe ser vista con cautela. Los datos del Censo de la Educación Superior (BRASIL 1995b) demuestran que, en 1995, año en que, teniendo en cuenta la edad promedia, los entrevistados (hombres y mujeres) estarían saliendo de la universidad, la

\footnotetext{
VIII Históricamente, el mercado de trabajo está segmentado en dos sentidos: horizontal (pocas profesiones y ocupaciones absorben la mayoría de las trabajadoras) y vertical (pocas mujeres en altos cargos, aún en los sectores con participación femenina preponderante como son la educación, la salud, la asistencia social, entre otros). En todas las culturas se realiza una interpretación bipolar (femenino - masculino) y a la vez jerárquica (lo masculino más valorizado que lo femenino) de las relaciones entre hombres y mujeres (YANNOULAS 2003).
}

Revista Zona Franca- Centro de estudios interdisciplinario sobre las mujeres (CEIM)- Maestría poder y sociedad desde la problemática de género (MG), Rosario, Argentina. ISSN, 2545-6504 http://zonafranca.unr.edu.ar/index.php/ZonaFrancal Numero 27 (2019). 
diferencia entre hombres y mujeres que concluían la carrera de Economía era menor que la participación de esos dos grupos en los cargos efectivos de la administración pública. Por tanto, ese argumento discursivo deja patente la fuerza de la imagen social de las mujeres como "poco objetivas", y menos interesadas o hábiles en trabajar con números, y al mismo tiempo muestra el uso de la ideología patriarcal para justificar la división sexual del trabajo (segmentación horizontal y vertical) vigente en la administración pública federal brasileña.

La articulación de lo simbólico con la base material surgió también en las ideas de maternidad de los entrevistados (hombres y mujeres) y actuaba fomentando la "auto-discriminación" y posibilitando la "discriminación encubierta" en la ocupación de altos cargos ${ }^{\mathrm{IX}}$.

Los relatos de las entrevistadas incluyen cambios en su área de actuación y en sus funciones laborales con el embarazo y la lactancia de los hijos, determinadas a partir del ideal de maternidad ${ }^{X}$ en el que fueron socializadas. La mayor parte de las entrevistadas que son madres afirmaron haber desistido de altos cargos cuando embarazadas o con hijos pequeños, porque pensaron que era lo correcto a hacer. Algunas de ellas también informaron no haber aceptado ocupar nuevos cargos para conciliar mejor trabajo remunerado y familia.

Entre los entrevistados no hubo relatos semejantes sobre diferencias establecidas en función del nacimiento de los hijos. Además, las "elecciones personales realizadas por las colegas", en el sentido de privilegiar la vida familiar

\footnotetext{
IX Yannoulas y Vallejos (1998) señalan la existencia de tres tipos de mecanismos de discriminación de sexo/género en las sociedades occidentales: la "discriminación manifiesta", que son reglas legitimadas y tienen el objetivo de salvaguardar los espacios de poder para los hombres; la "discriminación encubierta", que son códigos no explícitos que funcionan para garantir la inserción subordinada de las mujeres en el espacio productivo; y la "auto-discriminación", derivada de las dos anteriores, que constituye una especie de vigilancia que las mujeres internalizan para sentirse dentro de los parámetros establecidos por la sociedad.

${ }^{x}$ Biroli (2018) resalta que en las sociedades occidentales, las idealizaciones de familia y maternidad son productos de un mismo contexto histórico: el advenimiento de la burguesía como clase hegemónica. La separación de las esferas pública y privada, propia del capitalismo, permitió distinguir a la burguesía de otros estratos sociales y fue con esa separación que se configuraron la domesticidad femenina y el ideal de maternidad.
}

Revista Zona Franca- Centro de estudios interdisciplinario sobre las mujeres (CEIM)- Maestría poder y sociedad desde la problemática de género (MG), Rosario, Argentina. ISSN, 2545-6504 http://zonafranca.unr.edu.ar/index.php/ZonaFrancal Numero 27 (2019). 
en detrimento de la vida profesional, aparecen en los relatos de cinco de los entrevistados como fuerte argumento para demostrar que las mujeres no ocupan cargos más altos porque se "auto-discriminan" de ciertas funciones.

Sin embargo, los relatos de ellas dejan claro que no hubo apenas "autodiscriminación", o que ésta estaba anclada fuertemente en situaciones de discriminación encubierta. Las entrevistas dejan clara la existencia de un ideal profesional del servicio público, que coloca como incompatible la vivencia de la maternidad con la ocupación de altos cargos. Los relatos de ellos y de ellas fueron muy claros: o la maternidad acompañada de resignación cuanto a los ascensos o a la ocupación de altos cargos con mayor responsabilidad, prestigio y remuneración; o la aceptación de los cargos acompañada de una maternidad sacrificada o de una paternidad negligente.

La cuestión de la "conciliación" de demandas familiares y laborales permite discutir 3 aspectos de la segmentación vertical de la administración pública brasileña: (1) la organización del espacio público/productivo masculinizada; (2) la definición de un profesional ideal a partir de la experiencia masculina y; (3) la ocupación de DAS en la lógica del PDRAE, institucionalizando tal experiencia masculina.

Sobre el primer aspecto, es necesario tener en claro que esta división del espacio público/productivo y del espacio privado/reproductivo no es original ni exclusiva de la administración pública, pero que refuerza la actuación libre y sin impedimentos de algunos grupos sociales a partir de la existencia de otros grupos que asumen la responsabilidad por la atención de las necesidades de cuidado y reproducción humana y social, arcando con los costos que derivan de esa división. Según Biroli (2018), presumir que las mujeres asumen voluntariamente la función de cuidadoras, es decir, que consienten en la interpretación de que ellas son las que deben priorizar el cuidado, debería ser complementada por la explicación de las razones por las cuales la sociedad espera que apenas ellas asuman los costos

Revista Zona Franca- Centro de estudios interdisciplinario sobre las mujeres (CEIM)- Maestría poder y sociedad desde la problemática de género (MG), Rosario, Argentina. ISSN, 2545-6504 http://zonafranca.unr.edu.ar/index.php/ZonaFranca| Numero 27 (2019). 
o sacrificios que implica esa priorización. Al contrario de lo que afirmaron los entrevistados, la elección en si es una cuestión de género.

El segundo aspecto en discusión está intrínsecamente relacionado con el primero, porque es ese orden social que permite a los hombres dedicar largas horas al trabajo y a las actividades relacionadas con la profesión, tales como estudios y construcción de redes de contactos profesionales (BIROLI 2018). En ese contexto, el tiempo se transforma en factor clave, colocando a los hombres en situación de ventaja sobre las mujeres al competir en el mercado de trabajo en general y en el competitivo ambiente del servicio público federal en particular.

El tercer aspecto en discusión trata de la base generificada para las nociones de mérito y competencia que orientan la elaboración del Estado gerencial en el PDRAE. De esta manera, la idea de la existencia de un "mercado de DAS" en el cual los cargos en comisión en la administración pública federal brasileña serían ocupados por los profesionales de mayor mérito esconde el hecho de que la noción de mérito adoptada - de profesional autónomo, competitivo, esforzado y con el trabajo remunerado como valor central de su existencia - privilegia necesariamente a los hombres (BARBOSA 2003; BIROLI 2018). Al contrario de lo que sustentaba el PDRAE (BRASIL 1995), el "mercado de DAS" instituido en la administración pública federal brasileña tiende a premiar no a los profesionales más competentes, sino principalmente a aquellos con condiciones objetivas de dedicarse una mayor fracción de su día al trabajo remunerado.

En el caso de las entrevistadas, ofrecer disponibilidad de tiempo requerida por la ocupación de FCPE/DAS 4 y DAS 5 era posible porque no tenían hijos, o sus hijos ya eran grandes, o disponían de condiciones familiares extraordinarias como un marido asumiendo el cuidado de los niños pequeños; una red familiar ampliada que incluía madres, hermanas, suegras; o un superior jerárquico que, a partir de la vivencia de su propia paternidad, proporcionaba a sus subordinados horarios "más normales" de trabajo - que les permitían ajustar las tareas de

Revista Zona Franca- Centro de estudios interdisciplinario sobre las mujeres (CEIM)- Maestría poder y sociedad desde la problemática de género (MG), Rosario, Argentina. ISSN, 2545-6504 http://zonafranca.unr.edu.ar/index.php/ZonaFranca| Numero 27 (2019). 
cuidado de hijos pequeños a las demandas profesionales. Aun así, la posición estructural de estas trabajadoras era incómoda. Por un lado, presionadas a adecuarse al modelo masculino de dedicación al trabajo remunerado y, por otro, acusadas de abandonar el modelo femenino de dedicación a la familia.

Los arreglos de nuestras entrevistadas para conciliar trabajo remunerado y familia incluyen también la tercerización del trabajo de cuidados socialmente atribuido a ellas, conforme también descrito por Cyrino (2012) para las mujeres ejecutivas del sector privado del mercado de trabajo. La tercerización se efectúa a mujeres que ocupan la mayor parte del mercado de trabajo femenino o el polo opuesto de las mujeres trabajadoras (empleadas domésticas) (BRUSCHINI; LOMBARDI 2000). La delegación de tareas se relaciona principalmente con limpieza de la casa, lavar y planchar ropas, preparar alimentos para el consumo. El cuidado de hijos no era objeto de la tercerización, continua no siendo pago y es atribuido a otras mujeres del núcleo familiar. Arreglos semejantes realizan las familias de los hombres entrevistados, pero las mujeres que asumen el cuidado de hijos son sus esposas.

La inadecuación de las mujeres a un espacio de ejercicio profesional configurado según la experiencia masculina es una de las preocupaciones que aparecieron en las entrevistas. Ellas intentan copiar el comportamiento socialmente identificado con la masculinidad (objetividad, formalidad, etc.) para poder ser consideradas ocupantes potenciales de cargos de alto nivel en la jerarquía de la administración pública federal brasileña. El problema que se esconde en esta estrategia de las mujeres de adopción del "template masculino", expresión de Crompton y Le Fevre (SAVAGE; WITZ 1992), es que no elimina su condición de mujer. Por lo tanto, los efectos de la discriminación fueron constatados aun cuando las mujeres se comportaban como "hombres-substitutos" (SAVAGE; WITZ 1992). Para Savage y Witz (1992), en las organizaciones administrativas burocráticas, públicas y privadas, las mujeres son las facilitadoras, las limpiadoras, las ordenadoras, las apaciguadoras, las suavizadoras, las

Revista Zona Franca- Centro de estudios interdisciplinario sobre las mujeres (CEIM)- Maestría poder y sociedad desde la problemática de género (MG), Rosario, Argentina. ISSN, 2545-6504 http://zonafranca.unr.edu.ar/index.php/ZonaFranca| Numero 27 (2019). 
apoyadoras, entre otras funciones imprescindibles al capitalismo contemporáneo, para que los hombres puedan permanecer en el comando.

Lo más interesante del uso instrumental de las supuestas cualidades de las mujeres en las altas esferas de decisión del servicio público federal es que este uso no fue objeto de crítica por parte de todas las entrevistadas, ni constituyó propiamente un obstáculo para el desarrollo de las carreras de todas ellas. Una entrevistada inclusive resaltó que esa utilización de las características femeninas puede abrirles oportunidades a las mujeres, y otra, se negando a participar de las competiciones por cargos más altos de aquel ocupado por ella, demostró el profundo "realismo" que caracteriza frecuentemente la visión de mundo de los(las) dominados(as) (PUPPIM 1994). Ese profundo realismo de las mujeres, que no es resultado solamente de la percepción de su condición de inferioridad en el orden social patriarcal, sino también de la desigualdad de la propia competición establecida formalmente pero no intrínsecamente, puede ser interpretado no apenas como conformismo, sino también como resistencia (LEÓN 2002). Aun cuando sea una resistencia pasiva.

En ese contexto, el hecho de que 3 de los entrevistados hayan citado a la misma colega de carrera - que ocupaba la función de Secretaria Ejecutiva de un organismo federal - para demostrar como "hasta las mujeres" pueden alcanzar altos cargos en el comando de Estado sólo ratificaba la posición de "élite admitida" de las mujeres APO y EPPGG. Como recuerda León (2002), tal posición tiene dos consecuencias importantes. Primera, para aquellas mujeres no admitidas, la élite funciona como imagen social de que es posible llegar a ser Ministra, Presidenta, etc. Segunda, para aquellas mujeres admitidas, alimenta una idiosincrasia meritocrática poco solidaria, individualista: "yo llegué porque tengo características favorables para esto".

La preocupación relatada por las entrevistadas en adoptar el "template masculino" refleja también el temor a ser transformadas en "objeto sexual" por sus

Revista Zona Franca- Centro de estudios interdisciplinario sobre las mujeres (CEIM)- Maestría poder y sociedad desde la problemática de género (MG), Rosario, Argentina. ISSN, 2545-6504 http://zonafranca.unr.edu.ar/index.php/ZonaFrancal Numero 27 (2019). 
pares masculinos del servicio público federal. Pretenden cohibir eventuales investidas sexuales por parte de colegas de empleo. El temor al acoso sexual manifestado por las entrevistadas no parece injustificado. Dos de ellas relataron episodios de acoso sexual, y una confesó saber de un caso de otra colega. Dos entrevistados también afirmaron saber de estos casos. Siempre la víctima es una mujer y el agresor un hombre. En los 5 relatos oídos, el tipo más común fue el piropo, pero hubo también episodios más graves con contacto físico.

El acoso sexual constituye una de las muchas manifestaciones posibles de la discriminación sexual en el espacio laboral (CRUZ; YANNOULAS 2011). Tanto los casos de acoso sexual y moral como los comentarios que colocaban en duda la legitimidad de las conquistas profesionales de las mujeres, relatados por entrevistados y entrevistadas, contribuían para configurar un ambiente laboral hostil a la presencia femenina. Desde el punto de vista de Higa (2016), el mensaje que se pretende dar, aún de manera inconsciente, es el de que las mujeres no son bienvenidas allí y por lo tanto deberían permanecer en sus casas. El recelo a ser víctimas de conductas ilícitas de connotación sexual limitó al menos a 2 de las 7 entrevistadas a luchar por cargos en la alta jerarquía del servicio público federal. Dos de los entrevistados tienen la percepción de que el acoso sexual aparta a las mujeres de los altos cargos, y uno de ellos destacaba que era especialmente problemático para las más bonitas ${ }^{\mathrm{X}}$, cuando precisan quedarse hasta altas horas en el local de trabajo, o acompañar al jefe, lo que significaría riesgos potenciales mayores para las mujeres.

La hostilidad hacia ellas en un espacio profesional masculino por excelencia también se manifiesta en las dificultades para objetivar el liderazgo femenino, según relatos de 3 entrevistadas. La adopción de un estilo "femenino" (descrito

\footnotetext{
${ }^{\mathrm{XI}}$ La idea de que las mujeres consideradas bonitas corren más riesgos que otras mujeres se basa en la idea de que el acoso sexual es apenas el resultado del comportamiento lascivo de determinados hombres. Sin embargo, "la satisfacción de la lascivia mediante la extorsión no es el único propósito de los acosos sexuales, tal vez siquiera es el principal” (HIGA 2016:495). Muchas veces la intención del agresor es discriminar, mostrar poder, humillar.
}

Revista Zona Franca- Centro de estudios interdisciplinario sobre las mujeres (CEIM)- Maestría poder y sociedad desde la problemática de género (MG), Rosario, Argentina. ISSN, 2545-6504 http://zonafranca.unr.edu.ar/index.php/ZonaFranca| Numero 27 (2019). 
como democrático, horizontal, comprensivo, entre otras características señaladas por los entrevistados), despertaba renitencia; la adopción de un estilo "masculino" (autocrático, vertical, duro, entre otros aspectos apuntados), tampoco facilitaba el ejercicio de la autoridad por parte de las mujeres (EAGLY; CARLI 2007). Como dejó claro uno de los entrevistados, el estilo "masculino" de liderazgo en mujeres es desnecesario e indebido. Los relatos de los hombres revelaron que la adopción por hombres de un estilo femenino - "menos impositivo" y "más conciliador" - por su parte, no era considerado indebido o desnecesario. Las entrevistas mostraron que las dificultades de gestión enfrentadas pelas mujeres en altos puestos del Poder Ejecutivo federal se relacionan menos con el estilo de liderazgo y más con el hecho de que, en una sociedad patriarcal, la autoridad es vista como algo perteneciente al hombre (EAGLY; CARLI 2007; HIRATA et. al 2009).

En ese contexto, poco importa que el estilo femenino de conducción denominado liderazgo bottom-up (en que los subordinados participan de las decisiones); o el estilo interpersonally-oriented (que valoriza la manutención de relaciones interpersonales positivas con los subordinados); o inclusive el estilo transformational (en el cual la jefa se coloca como una mentora del equipo, estimulando a los subordinados a desarrollar sus potencialidades y así contribuir más efectivamente al alcance de las metas de la organización) ${ }^{X I I}$ - fuesen considerados muy positivamente en la época de la (re)funcionalización del Estado brasileño en la década de 1990 (EAGLY; CARLI 2007). O que ideas de transformación, revolución, reinvención y cambio de cultura impregnasen los textos sobre administración, gestión, programas de entrenamiento y cursos [Master in Business Administration -] MBA por los cuales los agentes habían pasado (NEWMAN; CLARKE 2012:360). Los cargos más altos de la jerarquía, aún

XII A esos estilos "femeninos" de liderazgo se oponen los estilos de liderazgo "masculinos", a saber: estilo de liderazgo top-down (en el cual la decisión es tomada de forma autocrática por el jefe); el estilo de liderazgo task-oriented (que enfatiza el cumplimiento de tareas por los subordinados); y el estilo de liderazgo transactional (el jefe establece apenas una relación de comando-control con sus subordinados (EAGLY; CARLI 2007).

Revista Zona Franca- Centro de estudios interdisciplinario sobre las mujeres (CEIM)- Maestría poder y sociedad desde la problemática de género (MG), Rosario, Argentina. ISSN, 2545-6504 http://zonafranca.unr.edu.ar/index.php/ZonaFrancal Numero 27 (2019). 
después de la reforma gerencial de 1995, continuaron a ser ocupados principalmente por hombres.

De hecho, las entrevistas realizadas confirman que en el gerenciamiento de Estado (neo/social-)liberal, como aquel propuesto en el PDRAE (BRASIL 1995) y vigente hasta 2017, la cuestión no es substituir un estilo "masculino" de liderazgo por uno "femenino", fundamentado en la valorización de los relacionamientos interpersonales, y si en usar un buen relacionamiento con los subordinados para lograr resultados demandados por la organización. No por acaso la mayoría de los hombres y mujeres entrevistados afirmó que gestionar personas constituía el principal desafío enfrentado en el cargo. Al decir de Antunes (2018), las técnicas contemporáneas de gestión de personas, con el involucramiento subjetivo de los trabajadores, son partes constitutivas de los "nuevos mecanismos de sujeción" capitalistas en tiempos de re-estructuración productiva y, así siendo, no deben ser tomadas como signos de una "feminização" de la gestión.

Por fin, pero no menos importante, constatamos que la naturalización de las desigualdades de sexo/género (y también de clase y raza/etnia) en la administración pública federal brasileña tuvo impacto en las políticas públicas que los entrevistados ayudaron a elaborar, implementar y monitorear. Nos llamó la atención el desconocimiento al respecto de las políticas públicas federales de enfrentamiento a las desigualdades en el ambiente de trabajo en curso, como el programa Pro-Equidad de Género y Raza ${ }^{X I I I}$, que fue mencionado y reconocido por apenas una entrevistada y un entrevistado. En general los entrevistados y entrevistadas cuestionaban el hecho de precisar políticas federales en este sentido, o la legitimidad del Estado para implementar ese tipo de acción. Abramo (2007), y Bandeira y Vasconcelos (2002), ya habían señalado anteriormente que

\footnotetext{
XIII El Programa Pro-Equidad de Género y Raza fue establecido en 2006 por la Secretaría Especial de Políticas para las Mujeres - SPM y su última edición fue realizada en 2016. El programa procuró diseminar nuevas maneras de gestión de personas y cultura organizacional para alcanzar la igualdad de género y raza en el local de trabajo. Fue direccionado a organizaciones públicas y privadas, de grande y medio porte (BRASIL 2016b).
}

Revista Zona Franca- Centro de estudios interdisciplinario sobre las mujeres (CEIM)- Maestría poder y sociedad desde la problemática de género (MG), Rosario, Argentina. ISSN, 2545-6504 http://zonafranca.unr.edu.ar/index.php/ZonaFrancal Numero 27 (2019). 
el conjunto de las nociones, percepciones e ideas que componen el imaginario de género de los agentes públicos contribuye, por acción o por omisión, para la (re)producción de las desigualdades de sexo/género en las propias políticas.

\section{A Modo de Conclusión}

Como mostraron las entrevistas realizadas en 2018 a 14 agentes públicos ocupantes de altos cargos en la administración pública brasileña, el ideal de profesional vigente está basado en la experiencia masculina, construido bajo el supuesto de que no apenas una separación entre los espacios público/productivo y privado/reproductivo es necesaria y saludable, cuanto en la "desresponsabilización" de los que actúan en la esfera pública con relación a la esfera privada. Así, el "mercado de los DAS" instituido por el PDRAE en ámbito federal en Brasil tiende a premiar a los hombres profesionales, con condiciones objetivas y subjetivas de dedicarse mayormente al trabajo remunerado.

Además, la investigación demostró que independientemente de la concepción de gerenciamiento en vigor a partir de los años 1990, la autoridad es vista como un atributo de los hombres. De esa forma, las dificultades enfrentadas por las mujeres en la ocupación de cargos de decisión de la administración pública federal brasileña no acaban cuando asumen la función o cargo en comisión. Inclusive las servidoras públicas federales que integran el "núcleo estratégico" del Estado - como las APO y EPPGG entrevistadas - enfrentan obstáculos para actuar en condiciones de igualdad frente a los hombres, especialmente a los subordinados.

Destruir las estructuras de (re)producción de las desigualdades de sexo/género (y también de clase y raza/etnia como por veces vislumbramos en los relatos de los entrevistados y las entrevistadas), no parece ser un asunto trivial. Involucra el reconocimiento de la necesidad de ir más allá de las políticas públicas, sin desconsiderar su importancia para las mujeres. El enfrentamiento de las

Revista Zona Franca- Centro de estudios interdisciplinario sobre las mujeres (CEIM)- Maestría poder y sociedad desde la problemática de género (MG), Rosario, Argentina. ISSN, 2545-6504 http://zonafranca.unr.edu.ar/index.php/ZonaFranca| Numero 27 (2019). 
desigualdades requiere mucho más que cambios en las pruebas de concurso público o la adopción de cuotas para mujeres y negros en los concursos, especialmente aquellos que llevan a carreras consideradas "núcleo estratégico" del Estado.

Como la ocupación de cargos efectivos en el servicio público federal desde el PDRAE se restringió a trabajadores con nivel de escolaridad superior, es preciso ampliar todavía más la participación de negros en la educación superior, y la participación de mujeres en los cursos que llevan a la ocupación de altos cargos, "tradicionalmente" masculinos. Al decir de Cappellin (2008), enfrentar no solamente el techo de cristal sino también las paredes de plomo!

Promover igualdad de género en altos cargos del servicio público federal, con el propósito de estimular la igualdad de género en las políticas formuladas, implementadas y monitoreadas por ellos, no se agota en la adopción de medidas de cuotas o en el cumplimiento de un horario estricto en el empleo, sin actividades fuera del horario de atención pública. Hay que avanzar en la responsabilización del Estado y de los hombres en la provisión social del cuidado, ampliando la oferta de servicios públicos para el cuidado de niños, ancianos y deficientes, licencia parental, etc., lo que permitirá que trabajadoras - públicas y privadas - dejen de sentir la doble presión por el desempeño perfecto en los dos espacios (MARCONDES 2012).

Por fin, la promoción de un ambiente laboral menos hostil a las mujeres exige la implementación de acciones de concientización y desnaturalización de la subordinación de las mujeres en el sistema patriarcal, y su relación con las prácticas discriminatorias en el espacio laboral. Inclusive, perfeccionar los mecanismos de denuncia, investigación y punición de tales prácticas en los organismos públicos federales brasileños.

\section{Bibliografía}

Revista Zona Franca- Centro de estudios interdisciplinario sobre las mujeres (CEIM)- Maestría poder y sociedad desde la problemática de género (MG), Rosario, Argentina. ISSN, 2545-6504 http://zonafranca.unr.edu.ar/index.php/ZonaFranca| Numero 27 (2019). 
ABRAMO, Laís (2007). "Inserção das Mulheres no Mercado de Trabalho na América Latina: Uma Força de Trabalho Secundária?" EnHIRATA, Helena; SEGNINI, Liliana (Org.). Organização Trabalho e Gênero (21-41). São Paulo: SENAC.

ANTUNES, Ricardo (2018). O Privilégio da Servidão - O Novo Proletariado de Serviços na Era Digital, Boitempo, São Paulo.

BANDEIRA, Lourdes; VASCONCELOS, Marcia (2002). Equidade de Gênero e Políticas Públicas: Reflexões Iniciais, AGENDE, Brasília.

BARBOSA, Livia (2003). Igualdade e Meritocracia - A Ética do Desempenho nas Sociedades Modernas, Editora FGV, Rio de Janeiro.

BARROSO, Carmen (2015). "As Mulheres nos Altos Escalões da Administração Pública no Brasil", enRevista do Serviço Público - RSP (RSP Revisitada), no 66 (1) 145-159 jan/mar, Brasília. Disponível em: shttp://repositorio.enap.gov.br/handle/1/2103>. Acesso em 12 jun. 2017.

BIROLI, Flávia (2018). Gênero e Desigualdades: Os Limites da Democracia no Brasil, Boitempo, São Paulo.

BRASIL. Ministério da Administração Federal e da Reforma do Estado MARE (1995). Plano Diretor de Reforma do Aparelho de Estado, MARE, Brasília. Disponível em: <https://edisciplinas.usp.br/pluginfile.php/192476/mod_resource/content/1/planodir etor\%20reforma\%20do\%20Estado\%20Bresser.pdf>. Acesso em 24 ago. 2017.

BRASIL. Ministério da Educação (1995b). Microdados do Censo Superior da Educação, S.I.. Disponível em: <httpp://portal.inep.gov.br?microdados>. Acesso em 05 nov. 2018.

BRASIL. Ministério da Transparência e Controladoria-Geral da União - CGU (2016a). Portal da Transparência, S. I. Disponível em: <httpp://www.portaltransparencia.gov.br/download-de-dados/servidores>. Acesso em 17 set.2018.

BRASIL. Secretaria Especial de Políticas para as Mulheres - SPM (2016b). Programa Pró-Equidade de Gênero e Raça - Rompendo Fronteiras no Mundo do

Revista Zona Franca- Centro de estudios interdisciplinario sobre las mujeres (CEIM)- Maestría poder y sociedad desde la problemática de género (MG), Rosario, Argentina. ISSN, 2545-6504 http://zonafranca.unr.edu.ar/index.php/ZonaFranca| Numero 27 (2019). 
Trabalho, SPM, Brasília. Disponível: http://www.onumujeres.org.br/wpcontent/uploads/2016/04/proequidade para-site.pdf. Acesso em: 24 dez. 2018.

BRASIL. Ministério do Planejamento, Desenvolvimento e Gestão - MP (2017). Boletim Estatístico de Pessoal e Informações Organizacionais- BEP, Vol.21 $\mathrm{n}$-249, MP, Brasília. Disponível em: shttp://www.planejamento.gov.br/assuntos/gestao-publica/arquivos-epublicacoes/BEP>. Acesso 01 jun. 2018.

BRUSCHINI, Cristina; LOMBARDI, Maria Rosa (2000). "A Bipolaridade do Trabalho Feminino no Brasil Contemporâneo". In:Cadernos de Pesquisa n 110 , 67-104, julho. Disponível em: <http://www.scielo.br/pdf/cp/n110/n110a03.pdf>. Acesso em 20 nov. 2017.

CAPPELLIN, Paola (2008). "As desigualdades impertinentes: telhado, paredes ou céu de chumbo?", en Gênero, v. 9, n. 1, p. 89-126.

CRUZ, Andréia; YANNOULAS, Silvia C. (2011), "Assédios Sexual e Moral no Trabalho. Um estudo acerca da legislação no Brasil”, enlbero Americana (Stockholm), v.XLI, p.137 - 170.

CYRINO, Rafaela (2012). Mulheres Executivas: A divisão do trabalho doméstico à luz dos estereótipos de gênero, Fino Traço, Belo Horizonte.

DEWES, João Osvaldo (2013). Amostragem em Bola de Neve e Respondent-DrivenSampling: Uma Descrição dos Métodos. Monografia (Bacharel em Estatística). Universidade Federal do Rio Grande do Sul, Porto Alegre/RS. Disponível em: <https://www.lume.ufrgs.br/bitstream/handle/10183/93246/000915046.pdf?sequen

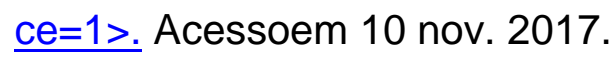

EAGLY, Alice H.; CARLI, Linda L. (2007). Through the Labyrinth: The Truth About How Womem Become Leaders, Harvard Business School Publishing Corporation, Boston.

HIGA, Flávio da Costa (2016). "Assédio Sexual no Trabalho e Discriminação de Gênero: Duas Faces da Mesma Moeda?",en Revista Direito GV, V. 12 №. 2, 484-515, mai-ago, São Paulo. Disponível em:

Revista Zona Franca- Centro de estudios interdisciplinario sobre las mujeres (CEIM)- Maestría poder y sociedad desde la problemática de género (MG), Rosario, Argentina. ISSN, 2545-6504 http://zonafranca.unr.edu.ar/index.php/ZonaFranca| Numero 27 (2019). 
<http://bibliotecadigital.fgv.br/ojs/index.php/revdireitogv/article/view/63633/61713>. Acesso em 24 nov. 2018.

HIRATA, Helena et al (Orgs.) (2009). Dicionário Crítico do Feminismo. Editora UNESP, São Paulo.

LÉON, MaríaAntonia García de (2002). Herederas y Heridas: Sobre lasÉlitesProfesionales Femininas, Ediciones Cátedra, Madrid.

MARCONDES, Mariana M. (2012)."A divisão sexual dos cuidados: do welfarestate ao neoliberalismo", enArgumentum, v. 4, n. 1, 91-107. Disponível em: shttp://periodicos.ufes.br/argumentum/article/view/2106>.

MINAYO, Maria Cecília de Souza (2008). O Desafio do Conhecimento: Pesquisa Qualitativa em Saúde. Hucitec Editora, São Paulo.

NEWMAN, Janet; CLARKE, John (2012). "Gerencialismo" enEduc. Real., v. 37, ํ. 2, 353-381, maio/ago, Porto Alegre. Disponível em: http://www.ufrgs.br/edu realidade. Acesso em 24 nov. 2018.

PUPPIM, Andréa Brandão (1994). "Mulheres em Cargos de Comando". En BRUSCHINI, Cristina; SORJ, Bila (Org.). Novos Olhares: Mujeres e Relações de Gênero no Brasil (13-35). São Paulo: Marco Zero: Fundação Carlos Chagas.

SAVAGE, Mike; WITZ, Anne (Ed.) (1992). Gender and Bureaucracy, Blackwell Publishers, Oxford.

YANNOULAS, Silvia Cristina (Coord.) (2003). A Convidada de Pedra: Mulheres e Políticas Públicas de Trabalho e Renda: Entre a Descentralização e a Integração Supranacional: Um Olhar a partir do Brasil (1988 - 2002), FLACSO, Editorial Abaré, Brasília.

YANNOULAS, Silvia Cristina (Coord.) (2013). Trabalhadoras: Análise da Feminização das Profissões e Ocupações. Editorial Abaré, Brasília.

YANNOULAS, Silvia Cristina; VALLEJOS, Adriana L (1998). "Elite de Mujeres: Elementos para la Compreensión de uma Expressión Particular de Discriminación" en La Aljaba - Revista de Estudios de la Mujer, vol III, 77-93.

Revista Zona Franca- Centro de estudios interdisciplinario sobre las mujeres (CEIM)- Maestría poder y sociedad desde la problemática de género (MG), Rosario, Argentina. ISSN, 2545-6504 http://zonafranca.unr.edu.ar/index.php/ZonaFrancal Numero 27 (2019). 